Historic, Archive Document

Do not assume content reflects current scientific knowledge, policies, or practices. 

Ounterbury Altroptipg

3ltrorputated

Eabinn, Allaryland
BOXWOOD

\section{RETAIL PRICE LIST}

FALL 1927 SPRING 1928

\section{FOR EWOR D}

The following wices include necessary packing and burlapping delivered in Easton, Maryland.

While every effort is made to mevent error in naming, and to deliver stock of the highest quality, no guarantee of any sort is made as to the description or vitality of any plants we sell. All sales are made and orders accepted only' with this distinet understanding.

Quotations are subject to change without notice, and stock unsold. Terms are strictly net eash on delivery.

The Nursery is prepared to render Landscape Service within Talbot County or near by.

Visitors are welcomed during office hours. We are closed Saturday afternoons and Sundays.

$$
\text { PHONE, EASTON } 30 F 2
$$




\section{DECIDUOUS TREES}

Name

Acer dasycarpum

Acer dasycarpum

Acer dasycarpum

Acer platanoides

Acer palmatum

Amygdalus persica,

Albizzir julibrissin

Albizzia julibrissin

Betula rubra

Catalpa speciosa

Celtis occidentalis

Cornus florida

Crataegus crusgalli

Crataegus tomentosa

Diospyros virginiana

Fraxinus viridis

Ginkgo biloba

Gleditschia sinensis

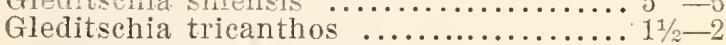

Koelreuteria paniculata ...........4 4 +5

Larix leptolepis $\ldots \ldots \ldots \ldots \ldots \ldots \ldots \ldots \ldots 5-6$

Miagnolia glauca ................... 3 ,

Malus $\ldots \ldots \ldots \ldots \ldots \ldots \ldots \ldots \ldots \ldots . .88=9$

Melia azedarach ......................

Photinia villosa ..................... 8

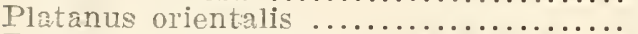

Populus alba bollerna

Populus nigra fastigiata

Populus nigra fastigiata

Salix babylonica

Salix babylonica

Salix niobe

Salix niobe

Sophora japonica

Taxodium distichum

Ulmus Americana

Zizjphus jujuba
Price $\mathrm{Tach}$

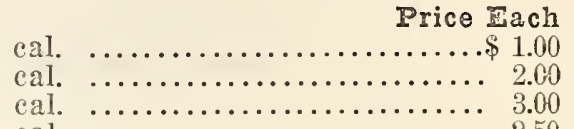

cal.

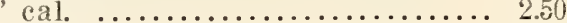

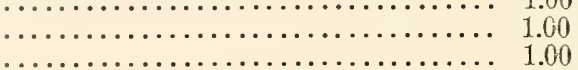

1.00
1.50

1.50
5.00

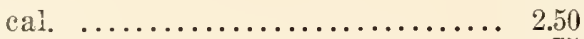

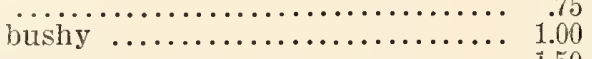

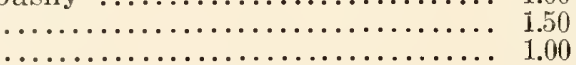

" cal. $\ldots \ldots \ldots \ldots \ldots \ldots \ldots \ldots \ldots \ldots, 1.50$

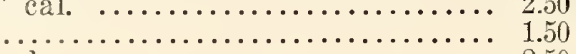

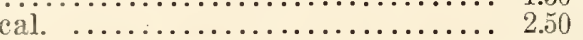

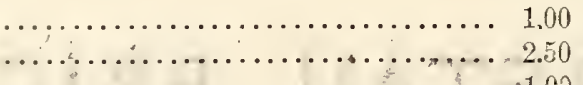

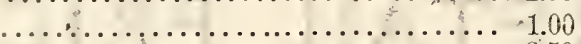

.......................... 3.50

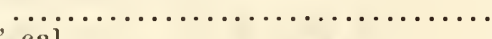

" cal.
1.00

.75
1.50

\section{Common Name}

Silver Míple

Silver Maple

Silver Maple

Norway Míple

Japanese Maple

Double Flowering Peach

Mimosa Tree

Mimosa Tree

Red Birch

Western Catalpa

Hackberry

Flowering Dogwood

Cockspur Hawthoin

Pear Hawthorn

Persimmon

Green Ash

Maidenhair Tree

Chinese Honeylocust

Honeylocust

Goldenrain Thee.

Japanese Lareh

Sweetbay

Flowering Crab

Chinaberry

1.50 Photinia

2.00 Oriental Plane

1.00 Bolleana Poplar

1.00 Lombardy Poplar

2.00 Lombardy Poplar

1.00 Weeping Willow

1.50 Weeping Willow

1.00 Golden Weeping Willow

2.00 Golden Weeping Willow

5.00 Chinese scholartree

2.00 Bald Cypress

3.50 American $\mathrm{Elm}$

2.50 Common Jujube

\section{DECIDUOUS SHRUBS}

Amygdalus nana, pink $\ldots . \ldots \ldots \ldots \ldots \ldots 2-3$

Azalea mollis ...........................

Beroeris ilicifolia $\ldots \ldots \ldots \ldots \ldots \ldots \ldots \ldots \ldots 1-11 / 4$

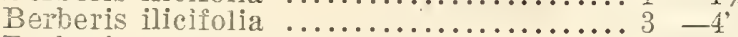

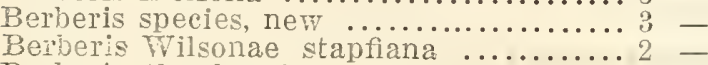

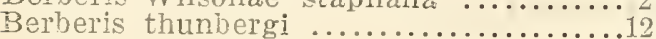

Berberis thunbergi

Beriberis thunbergi

Buddleia asiatica

Buddleia veichi

Callicarpa purpurea

Calycanthus floridu
Cercis canadensis

Cereis canadensis

Cercis japonica

Clethra alnifolia

Colutea arborescen

Cornus alba

Cornus sanguinea

Cornus sanguinea

Ceanothus americanu

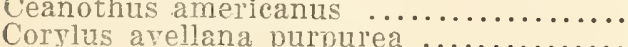

Cydonia japonica

Dentzia crenata

Dentzia gracilis

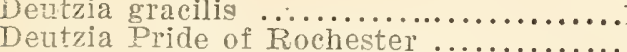

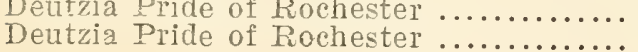

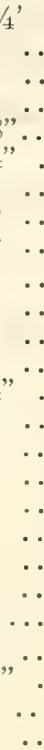

$: . \$$ 


\section{DECIDUOUS SHRUBS.-.Continued}

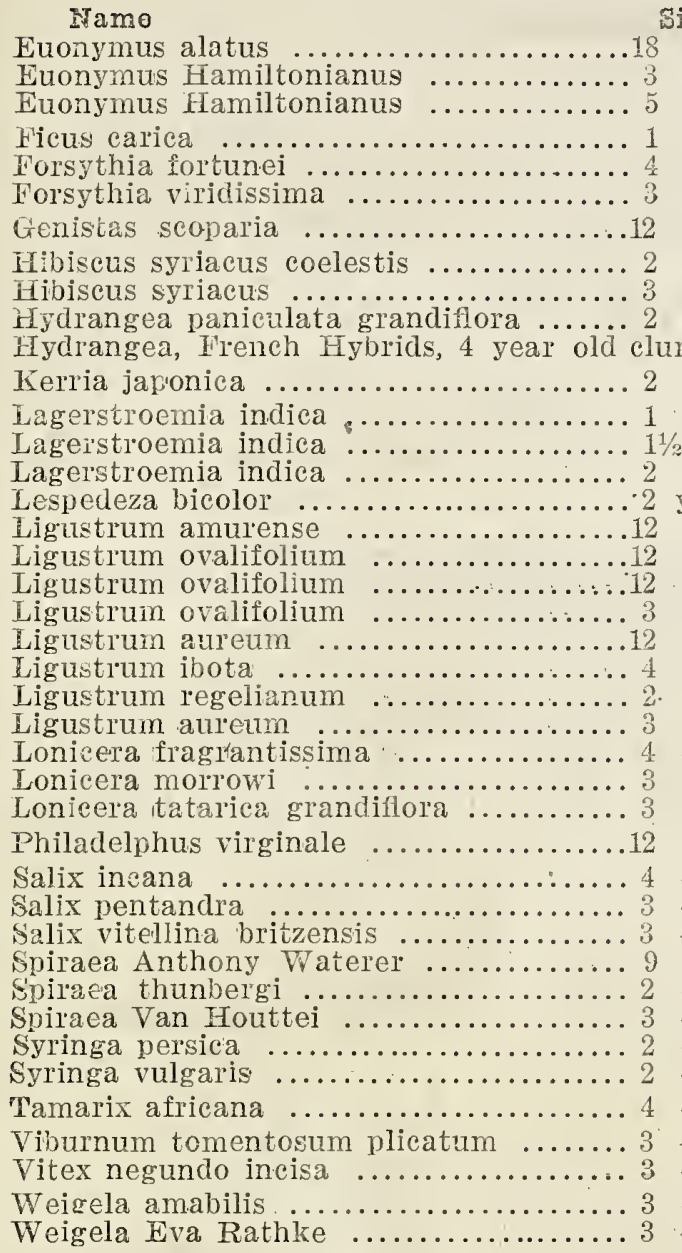

Sizo

Euonymus alatus $-24^{\prime \prime}$

$-4^{\prime}$,

Price Fach

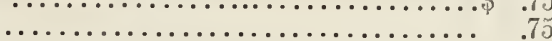

$\ldots \ldots \ldots \ldots \ldots \ldots \ldots \ldots \ldots \ldots \ldots \ldots \ldots, 1.00$

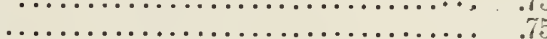

.

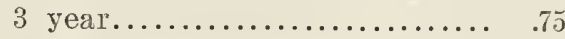

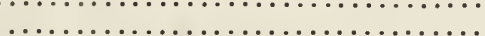

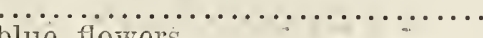

assorted colors

ascorted colors

assorted color's

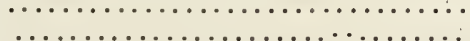

1 year

2 year

(n)

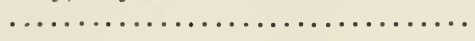

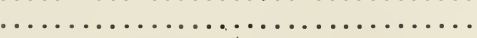

.

, n
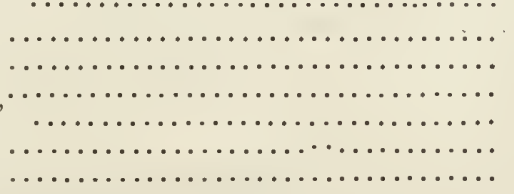

.

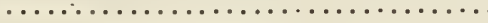

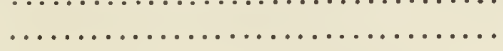

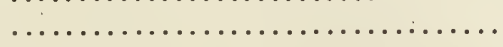

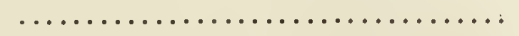

\section{EVERGREEN TREES}

Buxus arborescens

Buxus arborescens

Buxus arborescens

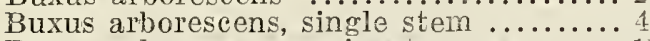

Buxus arborescens variegata ............. 11/2

Cedrus deodara

Chamaecyparis crippsi $\ldots \ldots \ldots \ldots \ldots \ldots \ldots \ldots \ldots$

Chamaecyparis Lawsoniana ...........

Chamaecyparis leptoclada

Chamaecyparis pisifera

Chamaecyparis plumosa

Chamaecyparis Rosedale

Cryptomeria japonica

Juniperus communis

Juninerus hibernica

Juniperus virginiana suecica

Juniperus virginiana

Juniperus virginiana glauca

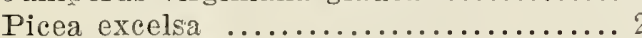

Pinus sylvestrig ...................

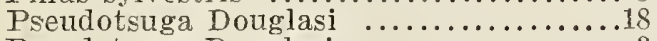

Pseudotsuga Douglasi $\ldots \ldots \ldots \ldots \ldots \ldots \ldots \ldots \ldots \ldots \ldots \ldots \ldots$

Thuya orientalis $\ldots \ldots \ldots \ldots \ldots \ldots \ldots \ldots \ldots$

Thuya orientalis aurea

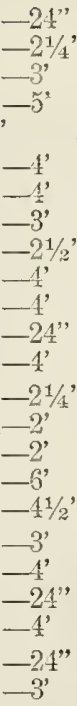

.$\$ 1.50$

2.50
3.50

5.00

1.00

5.00

4.00

2.50

5.00

3.50

3.00

2.50

3.50

3.50

1.50

1.00

500

6.00

2.00

3.00

2.00

3.00

2.50

2.50

\section{Common Name}

Winged Euonymus

Familton Euonymus

Hamilton Euonymus

Fig Bush or Tree

Purplestem Forsythia

Golden Bell

Scotch Broom

Blue Althaea

Double Red Althaea

Hydrangea

Japanese Hydrangeas

Kerria

Crepe Myrtle

Crepe Myrtle

Crepe Myrtle

Bush Clover

- Amur Privet

per hundred-Calif. Privet

per 100-Calif. Privet

per 100-Calif. Privet

\section{each-Golden Privet}

Ibota Privet

Regal Privet

Golden Privet

Fragrant Honejsuckle

Morrow Honeysuckle

Sweet Syringa

Rosemary Willow

Laurel Willow

Bronze Golden Willow

Spiraea

Thunberg Spiraea

Spiraea

Persian Lilac

Common Lilac

African Tamarix

Japanese Snowball

Chaste Shrub

Weigela Pink

Weigela Red
T'rue Tree Box

True Tree Box

True Box Trea

True Tree-Box

Variegated Tree Box

Deodara Cedar

Golden Cypress

Lawson Cypress

Andely Retinospora

Sawara Retinospora

Golden Plume Retinospora

Rosedale Hybrid

Common Cryptomeria

Common Juniper

Irish Juniper

Stredish Juniper

Red Cedar

Silver Redcedar

Norway Spruce

Scotch Pine

Douglas Fir

Douglas Fir

Oriental Arborvitae

Golden Oriental Arborvitae 


\section{EVERGREEN TREES---Continued}

Thuy oceidentalis ................2 $21 / 2-3$

Thula occicientalis lutea .............18 - 24 " "

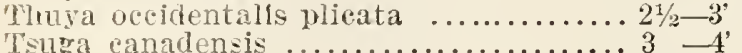

\section{EVERGREEN SHRUBS}

\begin{abstract}
dbelia grandifiora.
Abelia grandifiora

thelia grandifiola $\ldots \ldots \ldots \ldots \ldots \ldots \ldots \ldots$.

tzalea Hino di firi

Azalea Hino di Giri ...................

Burns Handsworthi ....................

Buxts janoniea .........................

Buxus jabonica .......................

Buxus sempervirens ................. 9 - 12"

Buxus sempervirens $\ldots \ldots \ldots \ldots \ldots \ldots \ldots \ldots \ldots .12 \quad-15$ "

Buxus sempervirens ...................... - 18 ",

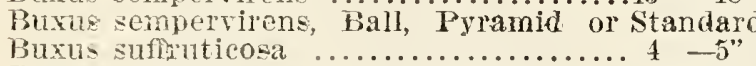

Buxus suffrutisosa $\ldots \ldots \ldots \ldots \ldots \ldots \ldots \ldots .5$. 6"

Burus suffuticosa ...............6 -

Buxus sufintuticosa ........................

Cunninghamia lanceolata ............15 - 18 "

Elaeagnis pungens ................

Euonymus acuta ..................... 18 x18"

Euonyms japonica .........................

Euonymus japoniea abo marginatus .....

Fuonyrus steboldi .................. 2

Jasminum mudiflorum .................

Juniperus Pftzeriana

Juniperus Pfitzeriana $\ldots \ldots \ldots \ldots \ldots \ldots \ldots \ldots . .3$.

Ligustrum amimense $\ldots \ldots \ldots \ldots \ldots \ldots \ldots 4 \quad-5$

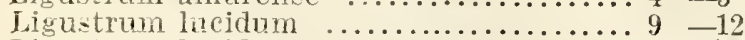

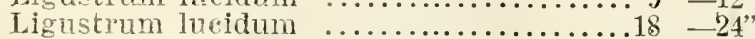

Ligustrum lucidum $\ldots . \ldots \ldots \ldots \ldots \ldots \ldots .2$.

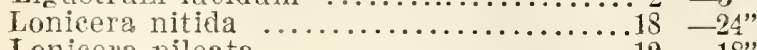

Lonicera pílenta ...........................

Mahonia aquifolia .................. -24 "

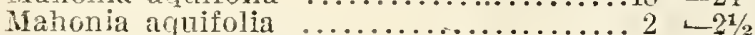

Osmanthus aquifolium ..................

Photinia serrulata $. . . \ldots \ldots \ldots \ldots \ldots . . .3-4$ '

Thuya orientalis nana aurea ........... 18 x18"

Thuya pumila ..................... 15 - 18 "

Viburnum rhytidophyllum ............18 - 24"
\end{abstract}

$\$ 2.50$ American Arborvitae

3.50 George Peabody Arborviate

2.50 Arborvitae

3.00 Canada Hemlock

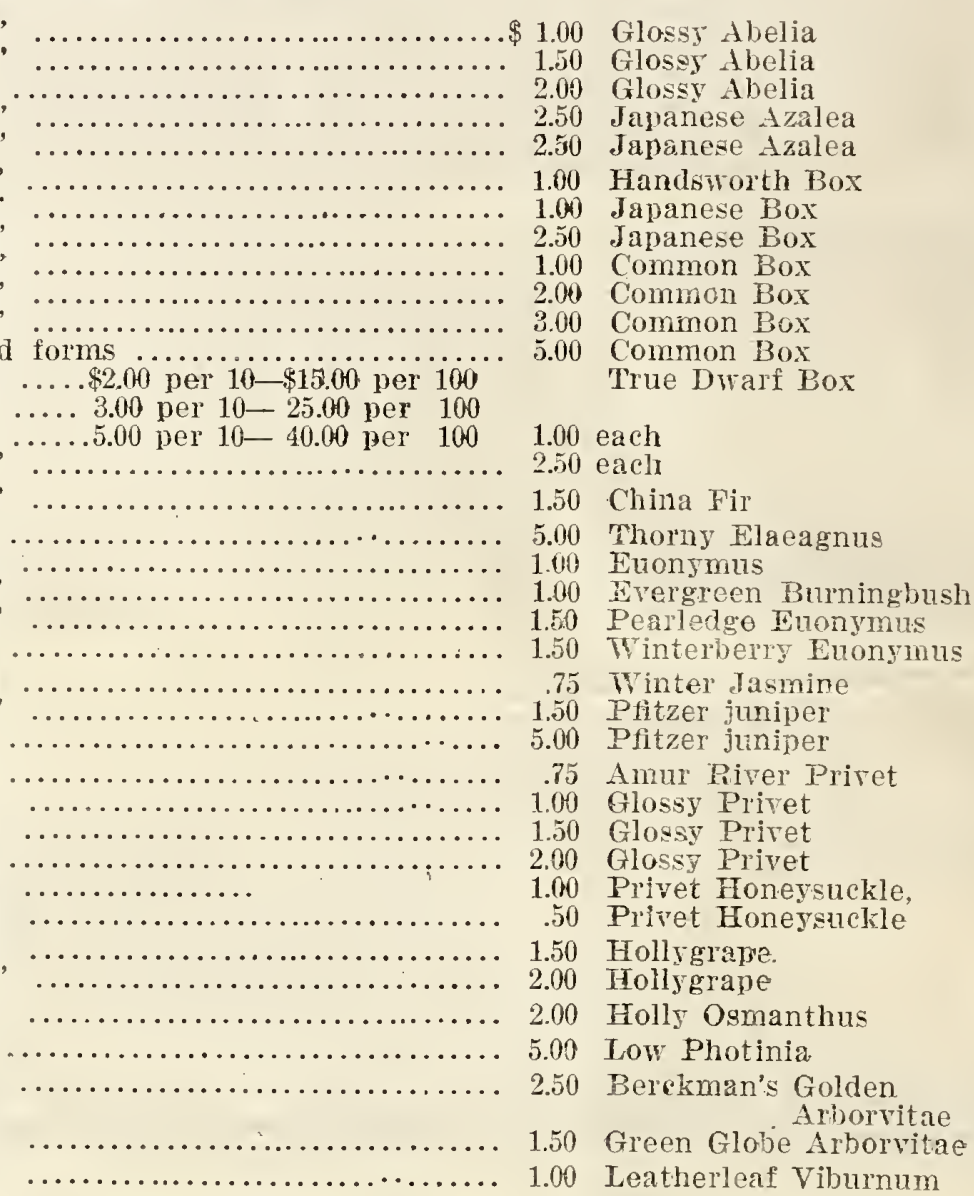

VINES

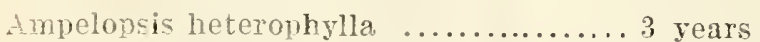
Enonyus radicans ................. 3 years

Hedera helix ..................... 2 years

Hedera helix in tubs trained in pyramid form

Iucca filamentosa $\ldots . . . \ldots \ldots \ldots . . .2$ year

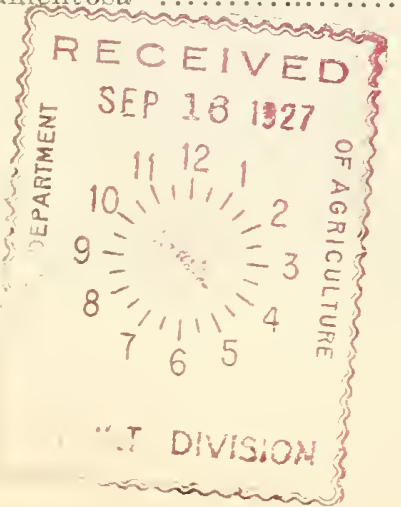

